\title{
Evidências Biológicas do Treino de Controle do Stress em Pacientes com Hipertensão
}

\author{
Biological Evidences of the Stress Management Training \\ in Patients with Hypertension
}

\author{
Lucia Emmanoel Novaes Malagris ${ }^{*}, a$, Tatiana Marlowe Cunha Brunini ${ }^{b}$, Monique Bandeira Moss ${ }^{b}$,

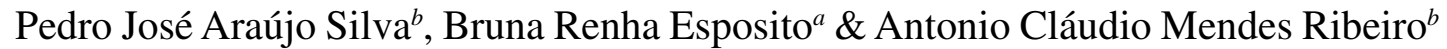 \\ ${ }^{a}$ Universidade Federal do Rio de Janeiro \& ${ }^{b}$ Universidade do Estado do Rio de Janeiro
}

\begin{abstract}
Resumo
O presente estudo objetivou avaliar o efeito do treino de controle de stress (TCS) em um grupo de pacientes hipertensas. Utilizou-se como critério para avaliação alterações na via L-arginina-óxido nítrico (NO). Participaram do estudo mulheres hipertensas $(n=44)$ e normotensas $(n=25)$, sendo que o TCS em grupo foi administrado em 14 hipertensas, observando-se as mudanças no nível de stress e no transporte da L-arginina. Em hipertensão, o transporte de L-arginina, através do sistema $\mathrm{y}^{+} \mathrm{L}$, mostrou-se reduzido, assim como o transporte de L-arginina pelos sistemas $\mathrm{y}^{+} \mathrm{L}$ e $\mathrm{y}^{+}$em pacientes hipertensas estressadas quando comparadas com normotensas estressadas. A redução do stress pelo TCS em hipertensas estressadas, restaurou o transporte de L-arginina através do sistema $\mathrm{y}^{+}$em níveis observados em pacientes hipertensas não-estressadas.

Palavras-chave: Stress; hipertensão; transporte celular.
\end{abstract}

\begin{abstract}
The objective of the present study was to evaluate the effect of stress management training in a group of hypertensive patients. Alterations of L-arginine-nitric oxide (NO) pathway were used as an evaluation criterion. Hypertensive $(n=44)$ and normotensive $(n=25)$ women participated in this study, and the stress management training was performed with a group of 14 hypertensive patients, observing the changes in the stress level and in L-arginine transport. In hypertension, the transport of L-arginine, via system $\mathrm{y}^{+}$, was reduced. Moreover, stressed hypertensive patients had a reduction of L-arginine transport by both systems, $\mathrm{y}^{+}$and $\mathrm{y}^{+} \mathrm{L}$, compared to stressed normotensive patients. The reduction of stress with stress management training in stressed hypertensive patients restored the transport of L-arginine via system $\mathrm{y}^{+}$to the same levels of non-stressed hypertensive patients.
\end{abstract}

Keyword: Stress; hypertension; cellular transport.

A hipertensão arterial sistêmica (HAS) é uma patologia com imensas repercussões sociais devido a sua morbidade e mortalidade, além de representar um fator de risco para doença cardiovascular (Sociedade Brasileira de Cardiologia, Sociedade Brasileira de Hipertensão, \& Sociedade Brasileira de Nefrologia [SBC, SBH, \& SBN], 2007). Ao mesmo tempo, vivemos em uma sociedade onde o stress se perpetua devido a estressores de todo o tipo, principalmente sociais (Dressler \& Santos, 2000; Lipp, 2005). Embora exista alguma evidência, não há uma comprovação efetiva de uma relação direta entre stress e hipertensão. As bases celulares e moleculares, tanto do stress como da hipertensão, só agora começam a ser investigadas em profundidade. Dentre as vias fisiopatológicas que, ao nível celular, participam da gênese e manutenção da hipertensão, destaca-se a via Larginina-óxido nítrico (NO) (Moss et al., 2004).

${ }^{*}$ Endereço para correspondência: Av. Pasteur, 250, Praia Vermelha, Rio de Janeiro, RJ, Brasil, CEP 22290-240. E-mail: lucianovaes@terra.com.br
A hipertensão arterial é uma enfermidade de etiologia multifatorial e mecanismos fisiopatológicos diversos. Os principais fatores de risco são a idade, o sexo, a etnia, os fatores socioeconômicos, o sal, a obesidade, o álcool e o sedentarismo (Carrol, Ring, Hunt, Ford, \& Macintyre, 2003; Dressler \& Santos, 2000; Harmann, Dias-da-Costa, Olinto, Pattussi, \& Tramontini, 2007). Convém ressaltar, que as V Diretrizes Brasileiras da Hipertensão Arterial (SBC, SBH, \& SBN, 2007) também fazem referência ao fator stress psicoemocional como responsável por elevação transitória da pressão arterial, assim como o stress mental. Mencionam estudos que evidenciam a influência do stress psicoemocional na reatividade cardiovascular e na pressão arterial assim como defendem a idéia de que a abordagem de aspectos psicoemocionais e psicossociais pode ser útil na melhora da adesão ao tratamento.

No Brasil, a hipertensão arterial é a doença mais freqüente na população adulta e que mais ocorre nos serviços de emergência. De acordo com as V Diretrizes Brasileiras de Hipertensão Arterial (SBC, SBH, \& SBN, 2007), no 
Brasil, estudos realizados em algumas cidades indicam prevalência de $22,30 \%$ a $43,9 \%$ da doença.

Fatores psicossociais como stress crônico, status socioeconômico, personalidade, depressão e suporte social estão relacionados com a ocorrência de elevação de pressão (Carrol et al., 2003; Dressler, \& Santos, 2000; Kivimaki et al., 2004; Straub, 2005). Esta associação está relacionada ao estilo de vida indicando que se deva investir em programas que alterem favoravelmente estes fatores (Strike \& Steptoe, 2004).

Stress pode ser definido como sendo: uma reação do organismo, com componentes físicos e/ ou psicológicos, causada pelas alterações psicofisiológicas que ocorrem quando a pessoa se confronta com uma situação que, de um modo ou de outro, a irrite, amedronte, excite ou confunda, ou mesmo que a faça imensamente feliz. (Lipp \& Malagris, 2001, p. 477).

A reação de stress envolve processos bioquímicos responsáveis por uma série de sintomas e sinais como, taquicardia, sudorese excessiva, hipervigilância, boca seca e tensão muscular. Tais sintomas típicos do início do processo de stress são semelhantes nas pessoas em geral. No entanto, à medida que o processo avança, sinais e sintomas específicos diferenciam-se, de acordo com a herança genética e possíveis vulnerabilidades desenvolvidas ao longo da vida de cada um (Lipp \& Malagris, 1995, 2001; Straub, 2005).

O processo do stress, de acordo com Selye (1965) ocorre em três fases: alarme ou alerta, resistência e exaustão, cada uma delas relacionada a reações específicas. Estudos recentes levaram ao conceito de uma nova fase do stress, entre a fase de resistência e a de exaustão, a quase-exaustão (Lipp, 2000, 2003a, 2004). A fase de quase-exaustão localiza-se ao final da fase de resistência de Selye, e propicia o desenvolvimento de patologias precedentes à exaustão (Lipp, 2003a, 2004; Malagris \& Fiorito, 2006).

A primeira fase do stress, a fase de alerta, está relacionada ao contato inicial do indivíduo com o estressor. A fase de resistência é a fase intermediária onde o organismo tenta restabelecer o equilíbrio interno, utilizando os mecanismos de adaptação, o que pode ser conseguido ou não. Quando o organismo não consegue voltar à homeostase, geralmente pela persistente presença do estressor, pode-se chegar à terceira (quase-exaustão) e à quarta fases (exaustão) do stress. É na fase de exaustão que costumam surgir as alterações patológicas associadas ao stress (Lipp, 2004; Lipp \& Malagris, 2001; Malagris, 2005). A reação de stress envolve um ou mais dos três eixos psicossomáticos: o neural, o neuroendócrino e o endócrino (Almeida, 2003; Everly, 1989; Leonard, 2005).

As alterações fisiopatológicas ocorridas em situações de stress excessivo envolvem, além de ativação neuro-hormonal, fenômenos inflamatórios na parede vascular, reatividade vascular aumentada, lesão vascular, ativação da cascata da coagulação e da trombogênese (Moreno, Melo, \& Rocha, 2003). A reatividade hipertensiva pode ser provocada tanto pelo stress mental (Pickering \& Gerin, 1990) como social (Stansfeld \& Marmot, 2002). O stress social relacionado à hipertensão ocorre na "hipertensão do jaleco branco" (Dean, Celis, Vandenhoven, O’Brien, \& Staessen, 2003; Lantelme \& Milon, 2000). Tal termo se refere ao fato de que alguns indivíduos têm sua pressão aumentada quando estão frente ao médico que vai aferi-la. Estudo realizado por Lipp, Pereira, Justo e Matos (2006) demonstrou que role-play de interações de conflito social pode estar associado a elevação de pressão. Tal estudo enfatiza que o conteúdo relacionado às situações de interações conflituosas experimentadas durante o role-play determina a magnitude da resposta.

A descoberta do óxido nítrico (NO), há mais de duas décadas, revolucionou o entendimento de importantes processos fisiológicos como a regulação do tônus vascular, da função plaquetária e do sistema imune (Moncada \& Higgs, 2006a). O NO é sintetizado a partir do aminoácido catiônico L-arginina por uma família de enzimas, as óxido nítrico sintases (NOS). Seu principal mecanismo de ação é através da ativação da guanilato ciclase que leva a um aumento da produção do GMPc intracelular (Moncada \& Higgs, 2006a). Vários estudos comprovam que a síntese de NO é dependente do transporte de L-arginina em células como endotélio, leucócitos e plaquetas (Brunini, Mendes-Ribeiro, Ellory, \& Mann, 2007) Em eritrócitos, foram descritos os sistemas $\mathrm{y}^{+}$e $\mathrm{y}^{+} \mathrm{L}$ (Devés \& Boyd, 1998; Verrey et al., 2004). A liberação inapropriada deste mediador ou uma reduzida disponibilidade de L-arginina tem sido associada com o stress e à patogênese de várias doenças, como a hipertensão arterial (Joint National Committee on Prevention, Detection, Evaluation and Treatment of High Blood Pressure, 2003; Moss et al., 2004; SBC, SBH, \& SBN, 2007). $\mathrm{O}$ completo entendimento e adequado tratamento destes estados patológicos permanece sendo um desafio.

Em pacientes hipertensos os resultados são controversos, mas a maior parte dos estudos indica que há uma diminuição na produção ou ação de NO na vasculatura (Cardillo \& Panza, 1998; Holm, Aukrust, \& Aagaard, 2002). Em contraste, outros estudos demonstraram que os níveis plasmáticos de nitritos e nitratos, metabólitos do NO, estão aumentados tanto em animais como em pacientes hipertensos (Dubei, Boegehold, Gillespie, Rosseli, \& Campase, 1996; Lauer, Kleinbongard, \& Kelm, 2002; Meireles et al., 2007; Vaziri, Ni, \& Oveisi, 1998).

Existe uma clara associação entre a síntese de NO e stress (Esch, Stefano, Fricchione, \& Benson, 2002; Eskiocak et al., 2006). A liberação de NO pelo hipotálamo modula o aumento da frequiência cardíaca e pressão arterial mediados pela noradrenalina em resposta ao stress (De Wardener, 2001; Hashiguchi, Ross-Cisneros, \& Alexander, 1997; Uji, Yoshida, Shintani-Ishida, \& Morimoto, 2007). Os níveis de metabólitos de NO aumentam no hipotálamo após a administração intra-peritoneal de citoquinas (Ishizuka et al., 2000). Como a resposta ao stress inclui um aumento na expressão de sistemas de transporte de L-arginina (Kakuda, Finley, Maruyama, \& MacLeod, 1998) que são também induzidos por citoquinas (Simmons, Closs, Cunningham, Smith, \& Kelly, 1996), é possível que a resposta ao stress 
envolva citoquinas, aumento de transporte de L-arginina e produção de NO em células chaves (Gulati, Ray, Masood, \& Vijayan, 2006; Moncada \& Higgs, 2006b) Uma alteração na disponibilidade intracelular de L-arginina pode ser o mecanismo comum entre as alterações da via L-argininaNO provocadas tanto pelo stress como pela hipertensão.

O NO modula a liberação dos hormônios do stress (ACTH e corticosterona), agindo como um facilitador da atividade ou liberação de neurotransmissores e mediando respostas imunes (Givalois, Li, \& Pelletier, 2002). Em ratos jovens, mas não idosos, o stress provocado por imobilização reduz os níveis plasmáticos de L-arginina. É possível que alterações nos níveis de L-arginina plasmática, durante ou após situações de stress, participem no desenvolvimento de patologias ou imunocompetência (Milakofsky, Harris, \& Vogel, 2000; Popovic, Zeh, \& Ochoa, 2007).

Até o momento, não existem relatos de estudos de transporte de L-arginina em hipertensão na presença de stress. Além disso, não se sabe os efeitos do treino de controle do stress sobre o transporte de L-arginina. O que parece claro é que o stress social leva a uma reatividade excessiva na pessoa hipertensa (Lipp et al., 2006) a qual pode ser reduzida com o tratamento do stress (Lipp, Bignotto, \& Alcino, 1997). O presente estudo teve como objetivo investigar aspectos relacionados à hipertensão essencial que ainda não foram propriamente esclarecidos: o papel da via L-argininaNO, especificamente alterações na disponibilidade de Larginina, e a contribuição do stress e do controle do stress nesta patologia.

\section{Método}

\section{Participantes}

O estudo envolveu 69 voluntárias do sexo feminino, 25 normotensas e 44 pacientes hipertensas nos estágios 1 e 2 . O grupo investigado foi constituído de pacientes e funcionárias normotensas do Hospital Universitário Pedro Ernesto (HUPE) da Universidade do Estado do Rio de Janeiro (UERJ). O estudo teve aprovação do Comitê de Ética e Pesquisa do Hospital Universitário Pedro Ernesto (processo 450-CEP/HUPE) e o consentimento por escrito de todos os participantes.

Foram critérios de inclusão/exclusão: ter a idade entre 30-65 anos, não usar drogas psicotrópicas, apresentar níveis glicêmicos normais, não ser portador de cardiopatia isquêmica, não ter sofrido acidente vascular cerebral, não sofrer de doença renal crônica ou doença mental grave e não ter sido transfundido nos últimos três meses.

\section{Instrumentos}

Foram utilizados como instrumentos, o Termo de Consentimento Livre e Esclarecido (TCLE), o Inventário de Sintomas de Stress para Adultos de Lipp (ISSL) (Lipp, 2000), uma entrevista semi-estruturada, o Manual do Treino de Controle do Stress de Lipp (TCS) (Lipp, 1991), assim como os materiais laboratoriais necessários para investigação do transporte de L-arginina em hemáceas.

\section{Procedimento}

Os participantes, normotensos e hipertensos, foram voluntários que, após receberem informações sobre o estudo e assinarem o TCLE, foram submetidos à entrevista psicológica visando obtenção de dados pessoais e de aspectos relacionados à hipertensão e verificação dos critérios de inclusão/exclusão. Nesse momento, foi avaliada presença e fase de stress, assim como sintomas (físicos e psicológicos) através do ISSL. A seguir, foi coletado sangue de todos aqueles que concordaram em participar do estudo, para que fosse possível encaminhar material para análise do transporte de L-arginina. Esse último foi realizado no Laboratório de Transporte de Membrana vinculado ao Departamento de Farmacologia e Psicobiologia do Centro Biomédico da UERJ.

Os pacientes e controles foram subdivididos em quatro grupos: normotensas estressadas, normotensas não-estressadas, pacientes hipertensas (estágios 1 e 2) estressadas e não-estressadas. Foi oferecida a possibilidade de participação no TCS para as hipertensas estressadas, no entanto, destas, apenas 14 se prontificaram a comparecer às 12 sessões que compõem o treino. Este subgrupo de pacientes hipertensas estressadas participou do TCS de Lipp e as demais, no momento da entrevista, receberam orientações resumidas de como lidar com o stress e de locais onde buscar atendimento psicológico gratuito. Foram consideradas para pareamento estatístico as seguintes variáveis: nível de pressão arterial, idade, índice de massa corporal e presença ou não de stress.

\section{O Treino de Controle do Stress (TCS) de Lipp}

O TCS se constitui em um protocolo de tratamento do stress de base cognitivo-comportamental desenvolvido por Lipp que se constitui em 12 sessões voltadas para atuação em quatro áreas buscando mudança de estilo de vida e, consequentemente, controle do stress apresentado. As áreas citadas se constituem em orientação nutricional anti-stress, orientação sobre atividade física, treino de técnicas de relaxamento e respiração profunda e intervenção frente a aspectos psicológicos que podem se constituir em fontes internas de stress (Lipp, 2003b; Lipp \& Malagris, 1995, 2001).

\section{Coleta de Sangue e Transporte de L-Arginina em Eritrócitos}

Foram coletados $10 \mathrm{ml}$ sangue de controles e pacientes hipertensos através de venopunção, usando-se heparina como anticoagulante.

Os influxos de L-arginina foram realizados de acordo com um protocolo estabelecido há 10 anos (Mendes Ribeiro et al., 1997). Os eritrócitos são isolados por centrifugação. N-etil-maleimida (NEM) é usada para isolamento dos sistemas de transporte $\mathrm{y}^{+}$e $\mathrm{y}^{+} \mathrm{L}$. A seguir, Larginina tritiada $(5-500 \mu \mathrm{M})$ é adicionada à suspensão de eritrócitos, incubada a $37^{\circ} \mathrm{C}$. O sangue é então resfriado e lavado com $\mathrm{MgCl}$., as células lisadas e as proteínas precipitadas com TCA. Após centrifugação, o sobrenadante é retirado, líquido cintilante adicionado e a radioativida- 
Malagris, L. M. N., Brunini, T. M. C., Moss, M. B., Silva, P. J. A., Esposito, B. R. \& Ribeiro, A. C. M. (2009). Evidências Biológicas do Treino de Controle do Stress em Pacientes com Hipertensão.

de contada. Todos os produtos químicos foram adquiridos dos fabrican-tes Sigma, Poole, Dorset, UK.

\section{Análise Estatística}

Os dados encontrados no ISSL foram analisados através de estatística descritiva utilizando-se cálculo de porcentagens. A significância estatística foi considerada quando $p<0.05$ e o cálculo de médias incluiu erro padrão (EP). O $\mathrm{V}_{\max }$ e $\mathrm{K}_{\mathrm{m}}$ dos sistemas de transporte, bem como a difusão, foram determinados através da equação Michaelis-Menten, utilizando o programa Enzfitter (Elsevier). Os dados experimentais foram analisados pelo teste não paramétrico Mann-Whitney (prova $U$ ) para comparar grupos dois a dois e teste $t$ de Student pareado para comparar o mesmo grupo antes e depois do TCS. Os cálculos estatísticos e o preparo das figuras foram realizados através do programa de computador GraphPad Prism 3.0.

Tabela 1

Presença de Stress nas Participantes Normotensas e Hipertensas

\begin{tabular}{lcccccc}
\hline \multirow{2}{*}{ Stress } & \multicolumn{2}{c}{ Normotensas } & \multicolumn{2}{c}{ Hipertensas } & \multicolumn{2}{c}{ Total } \\
& Freqüência & $\%$ & Frequiência & $\%$ & Freqüência & $\%$ \\
\hline Estressadas & 12 & 48 & 32 & $72.7 *$ & 44 & 63.7 \\
Não-estressadas & 13 & 52 & 12 & 27.3 & 25 & 36.3 \\
\hline Total & 25 & 100 & 44 & 100 & 69 & 100 \\
\hline
\end{tabular}

Nota. $* p<0.05$.

O ISSL revelou que 10 (83.3\%) das normotensas estressadas se encontravam na fase de resistência do stress, uma participante na fase de quase-exaustão e uma na fase de exaustão. Nenhuma participante estava na fase do alerta. Dentre as que se encontravam em fase de resistência, 90\% apresentaram mais sintomas psicológicos como manifestação do stress. Da mesma forma, as normotensas que se encontravam na fase de quase-exaustão (1) e de exaustão (1) apresentaram também mais sintomas psicológicos.

Quanto às hipertensas, $21(65.6 \%)$ pacientes estavam na fase de resistência do stress, oito (25\%) na fase de quaseexaustão, duas na fase de exaustão e uma na fase do alerta. Dentre as pacientes hipertensas que estavam na fase de resistência, $13(61.9 \%)$ apresentaram mais sintomas psicológicos na manifestação do stress, cinco (23\%) apresentaram mais sintomas físicos e três (14.3\%) os dois tipos de sintoma concomitantemente. A única paciente na fase de alerta também teve predominância de sintomas psicológicos. Oito $(25 \%)$ pacientes hipertensas estavam na fase de quase-exaustão. Dessas, quatro (50\%) apresentavam mais sintomas psicológicos, três $(37.5 \%)$ mais sintomas físicos e uma paciente os dois tipos concomitantemente. As duas pacientes em exaustão revelaram mais sintomas psicológicos.

A Tabela 2 descreve as fases do stress das 14 pacientes que constituíram o grupo de hipertensas que participou do TCS. Antes do TCS, 10 (71.4\%) estavam na fase de resistência, três (21.4\%) na fase de quase-exaustão, uma em exaustão e nenhuma em alerta. Após o TCS das 14 estressadas, apenas quatro (28.5\%), previamente na fase de resistência, se mantiveram estressadas no mesmo nível de stress. Em relação aos sintomas associados ao stress, antes do TCS, entre as 10 pacientes hipertensas que estavam na fase de resistência, sete (70\%) apresentaram mais sintomas psicológicos como manifestação do stress. Entre as três pacientes hipertensas que estavam na quase-exaustão duas tinham mais sintomas físicos e a paciente que estava na exaustão tinha mais sintomas psicológicos. Após o TCS, as quatro pacientes hipertensas que continuaram estressadas na fase de resistência, apresentaram mais sintomas psicológicos. De forma interessante, as pacientes hipertensas que estavam em fase de quase-exaustão (3) e exaustão (1) não apresentaram stress após o TCS (Tabela 2).

Tabela 2

Fases de Stress em Hipertensas Estressadas Antes e Depois do Treino de Controle do Stress (TCS)

\begin{tabular}{lcccc}
\hline Fases & \multicolumn{2}{c}{ Pré - TCS } & \multicolumn{2}{c}{ Pós - TCS } \\
& Freqüência & Freqüência & $\%$ \\
\hline Alerta & - & - & - & - \\
Resistência & 10 & $71.4 \%$ & 42 & $8.5 \%$ \\
Quase-Exaustão & 3 & $21.4 \%$ & - & - \\
Exaustão & 1 & $7.2 \%$ & - & - \\
\hline Total & 14 & $100 \%$ & 4 & $28.5 \%$
\end{tabular}


Quanto aos resultados referentes ao transporte de L-arginina, considerando o fator stress, o transporte de L-arginina pelos sistemas $\mathrm{y}^{+}$e $\mathrm{y}^{+} \mathrm{L}$ em eritrócitos estava significativamente reduzido em pacientes hipertensas estressadas comparado com normotensas estressadas conforme mostra as Figuras 1 e 2.
O transporte de L-arginina, através do sistema $\mathrm{y}^{+}$no subgrupo de hipertensas estressadas que participaram do TCS aumentou após o treino de controle de stress. Em contraste, o sistema $y^{+} \mathrm{L}$ não foi afetado pela intervenção como pode ser visto na Figura 3.

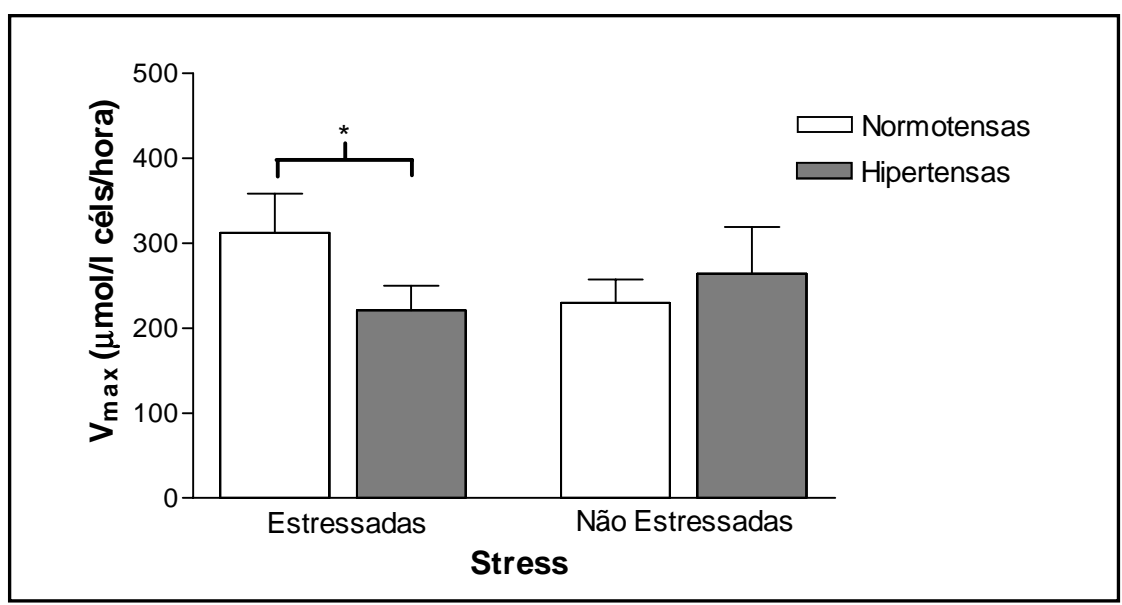

Figura 1. Transporte celular de L-arginina em eritrócitos pelo sistema $\mathrm{y}^{+}$em hipertensas e normotensas de acordo com o stress. Nota. ${ }^{*} p<0.05$.

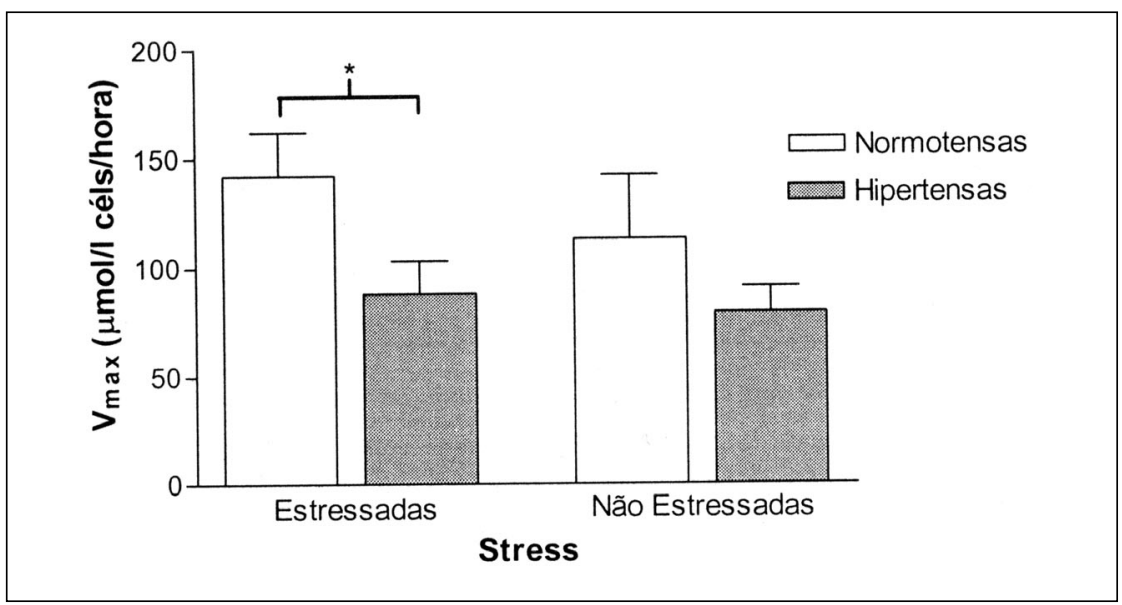

Figura 2. Transporte celular de L-arginina em eritrócitos pelo sistema $\mathrm{y}^{+} \mathrm{L}$ em hipertensas e normotensas de acordo com o stress. Nota. $* p<0.05$.

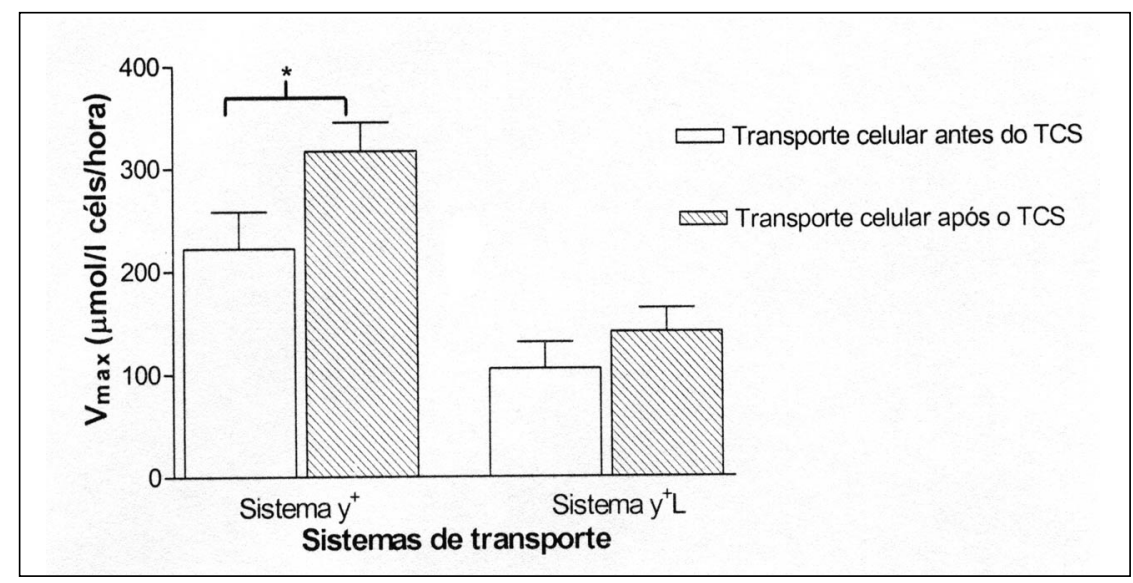

Figura 3. Transporte celular de L-arginina pelos sistemas $\mathrm{y}^{+}$e $\mathrm{y}^{+} \mathrm{L}$ em hipertensas estressadas antes e após o Treino de Controle do Stress (TCS). Nota. ${ }^{*} p<0.05$. 
Malagris, L. M. N., Brunini, T. M. C., Moss, M. B., Silva, P. J. A., Esposito, B. R. \& Ribeiro, A. C. M. (2009). Evidências Biológicas do Treino de Controle do Stress em Pacientes com Hipertensão.

\section{Discussão}

Os dados encontrados no presente estudo indicaram que o Treino de Controle do Stress de Lipp (Lipp, 1991), protocolo de tratamento baseado na abordagem psicoterapêutica intitulada cognitivo-comportamental, foi efetivo no que se refere a produzir alterações biológicos favoráveis ao nível de transporte celular do aminoácido L-arginina. Observou-se que o tratamento psicológico aplicado ao grupo de 14 hipertensas estressadas gerou aumento no transporte do aminoácido L-arginina, precursor do NO, em eritrócitos. Se esta ativação do transporte de L-arginina relatada neste estudo for um fenômeno universal, outras células como endotélio e leucócitos, podem estar associadas com a produção aumentada de NO após o Treino de Controle do Stress de Lipp.

Considerando a importância desse aminoácido para a produção do NO, gás vasodilatador e anti-agregante plaquetário que se mostra reduzido em sua biodisponibilidade em hipertensos (Cardillo \& Panza, 1998; Holm et al., 2002; Meireles et al., 2007), pode-se concluir que o TCS se constitui em um importante tratamento coadjuvante ao tratamento medicamentoso para hipertensos.

Os resultados encontrados também no presente estudo quanto à redução do transporte do aminoácido Larginina pelos dois sistemas de transporte $\left(\mathrm{y}^{+}\right.$e $\left.\mathrm{y}^{+} \mathrm{L}\right)$ em hipertensas estressadas quando comparadas com normotensas estressadas, já estariam indicando a importância do stress na redução do transporte pelo sistema $\mathrm{y}^{+}$. Isto porque estudo anterior (Moss et al., 2004) já havia indicado a redução do transporte em hipertensos apenas pelo sistema $\mathrm{y}^{+} \mathrm{L}$ e não pelo $\mathrm{y}^{+}$. Ou seja, se na hipertensão encontrou-se redução do transporte de L-arginina pelo sistema $\mathrm{y}^{+} \mathrm{L}$ e quando se introduz o stress como variável se encontra redução pelos dois sistemas, parece coerente pensar-se no stress como interferindo, especialmente, no transporte pelo sistema y+.

Observa-se que, tanto quando se considera apenas a hipertensão como quando se considera hipertensão e stress concomitantemente, encontrou-se redução do transporte, mas parece que cada variável é responsável por tal redução através de um sistema.

Resumindo, estudo anterior acima citado concluiu que é possível que a diminuição da atividade de transporte de L-arginina através do sistema $\mathrm{y}^{+} \mathrm{L}$, se for um fenômeno universal, seja responsável pela redução da concentração da L-arginina intracelular limitando a produção de NO em hipertensão. O presente estudo, que introduziu a variável stress, concluiu que quando o stress está presente em hipertensos, esse transporte se reduz ainda mais, o que, possivelmente, gera diminuição ainda maior na produção de NO, trazendo prejuízos ainda maiores para os hipertensos.

Embora, estudos anteriores já comprovassem que o stress afeta a via L-arginina-NO (De Wardener, 2001; Esch et al., 2002; Eskiocak et al., 2006; Givalois et al., 2002; Hashiguchi et al., 1997; Ishizuka et al., 2000; Kakuda et al., 1998; Milakofsky et al., 2000; Uji et al., 2007), o presente estudo associa hipertensão, stress, treino de controle do stress e alterações de transporte de L-arginina, não havendo relatos, até o momento, de estudos considerando todas essas variáveis concomitantemente.

Os resultados encontrados no presente estudo parecem constituir-se importante contribuição no estudo da hipertensão, já que embora esta patologia venha sendo investigada intensamente, ainda paira sob a mesma questionamentos quanto a sua gênese e os exatos mecanismos envolvidos. $\mathrm{O}$ fato de ser uma síndrome clínica multifatorial exige que pesquisas sejam realizadas buscando investigar a forma de influência dos diversos fatores envolvidos na doença, e a via L-arginina-NO se constitui em um desses fatores. Isto porque o NO regula o fluxo sanguíneo renal e o volume plasmático, função cardíaca e o tônus vascular (Esch et al., 2002; Rudd, Trolliet, \& Loscalzo, 2000) e a sua inibição pelo L-NAME pode levar à hipertensão (Holm et al., 2002; Klahr, 2001; Moncada \& Higgs, 2006a; Rudd et al., 2000; Taddei et al., 1996).

Outro fator envolvido na hipertensão é o stress, o qual vem sendo cada vez mais foco de estudos detalhados visando entender essa associação (Dean et al., 2003; Lantelme \& Milon, 2000; Lipp et al., 2006; Moreno et al., 2003; Pickering \& Gerin, 1990; Stansfeld \& Marmot, 2002). O fato de se concluir sobre alterações no transporte celular de L-arginina no stress e em seu tratamento psicológico, além de ser um aprofundamento no estudo da relação do stress com a via L-arginina-NO, também, se constitui em um avanço no entendimento da associação entre stress e hipertensão. O presente estudo demonstra que o stress e a hipertensão estão interligados através dessa via.

Relevante se torna questionar até que ponto fatores psicossociais podem levar a alterações celulares em pacientes hipertensos. A resposta óbvia é que, se estes fatores são capazes de induzir, manter e agravar a hipertensão, assim o fazem modulando estruturas celulares e moleculares.

Pode-se, então, estabelecer um conjunto de fatores interligados que poderiam aumentar o risco de uma pessoa tornar-se hipertensa. A lista destes fatores de risco inclui elementos biológicos, genéticos, nutricionais, psicológicos e sociais.

A importância de elementos psicológicos no desenvolvimento e manutenção da hipertensão arterial já é um fato aceito. Os resultados aqui obtidos quanto à avaliação do stress nas participantes do estudo revelaram que haviam mais hipertensas estressadas do que normotensas, o que confirma a relação do stress com a hipertensão. Observase que o stress participa na fisiopatologia da hipertensão e, possivelmente, esta irá agravar o próprio stress, já que se desenvolve um círculo vicioso. Assim, preocupações com as conseqüências da doença, com a medicação, o desconforto, o medo da morte, podem tornar a vida do hipertenso mais estressante. Tal fato pode ser confirmado através do dado aqui encontrado quanto à manifestação sintomatológica do stress predominante entre as participantes, que foi psicológica e não física.

A fase do stress predominante encontrada entre as participantes estressadas foi a de resistência, tanto nas normotensas como nas hipertensas, o que sugere que estavam 
estressadas já há algum tempo e que já estaria havendo um comprometimento dos sistemas neural, neuro-endócrino e endócrino (Everly, 1989).

Como visto anteriormente, na primeira fase do stress (alerta), embora já haja uma quebra na homeostase do organismo, o desgaste ainda não é muito acentuado, enquanto a fase de resistência envolve uma tentativa do organismo de recuperar seu equilíbrio (Everly, 1989; Lipp \& Malagris, 2001). A fase de alerta está associada à ativação do sistema nervoso autônomo e da medula da supra-renal gerando a reação de "luta ou fuga" (Selye, 1965). Já a fase de resistência está associada ao início do processo de "carga alostática" que se refere à falha do organismo em manter a homeostase (McEwen, 2003), envolvendo o córtex da supra-renal e a produção de corticosteróides, como anteriormente descrito. $\mathrm{O}$ processo de resistência quando não resolvido com sucesso leva ao desgaste de múltiplos sistemas fisiológicos, contribuindo para o desenvolvimento de patologias comuns na terceira (quase-exaustão) e na quarta fase (exaustão) do stress. Dentre tais patologias, encontra-se a hipertensão.

A partir da compreensão do processo do stress através das fases, torna-se mais fácil entender as alterações fisiológicas diversas da fase de resistência, o que, aqui, se mostrou através da alteração na via L-arginina-NO.

Importante também se faz refletir sobre a avaliação do stress após a participação no TCS a qual revelou que das 14 pacientes hipertensas estressadas, $71.4 \%$ deixaram de apresentar stress e $28.5 \%$ continuaram estressadas permanecendo na mesma fase do stress (resistência). As pacientes que deixaram de apresentar stress após o TCS, antes do mesmo estavam: seis na fase de resistência, três em quaseexaustão e uma em exaustão. Convém ressaltar, que algumas participantes foram mais disciplinadas na freqüência às sessões e na realização das tarefas solicitadas, o que pode explicar o resultado encontrado. As estratégias ensinadas visam mudança no estilo de vida e, sendo introduzidas pelo hipertenso, podem ser importantes no controle da doença. Dentre estas se incluem técnicas de respiração profunda e relaxamento, além de estratégias cognitivas que procuram gerar uma forma realista e adaptativa de interpretar e lidar com os eventos do dia-a-dia. Dessa forma, procura-se uma redução no número e intensidade de estressores e uma conseqüente desativação fisiológica comum em situações de stress e na hipertensão.

Concluindo, em pacientes hipertensas estressadas, o transporte de L-arginina pelos sistemas $\mathrm{y}^{+}$e $\mathrm{y}^{+} \mathrm{L}$ em eritrócitos estava significativamente reduzido. Após o treino de controle do stress, o qual reduziu o número de pacientes hipertensas estressadas significativamente, o transporte de L-arginina através do sistema de alta capacidade $\mathrm{y}^{+}$, e não pelo $\mathrm{y}^{+} \mathrm{L}$, foi aumentado a valores semelhantes aos obtidos com as pacientes inicialmente não-estressadas da amostra. Os resultados obtidos mostram que o stress reduziu o transporte de L-arginina em eritrócitos das hipertensas estressadas do presente estudo e que o TCS de Lipp recuperou esse transporte aos níveis semelhantes aos das hipertensas não estressadas. Tais conclusões podem levar a reflexão de que, a partir da restauração do transporte de L-arginina, possa ocorrer um aumento da produção do NO nesses pacientes, o que pode contribuir para o controle da pressão arterial em conjunto com o tratamento medicamentoso.

\section{Referências}

Almeida, O. M. M. S. (2003). A resposta neurofisiológica ao stress. In M. Lipp (Ed.), Mecanismos neuropsicofisiológicos do stress: Teoria e aplicações clínicas (pp. 26-30) São Paulo, SP: Casa do Psicólogo.

Brunini, T. M. C., Mendes-Ribeiro, A. C., Ellory, J. C., \& Mann, G. E. (2007). Platelet nitric oxide synthesis and activation in uremia and malnutrition: A role for L-arginine supplementation in vascular protection? Cardiovascular Research, 73, 359-367.

Cardillo, C., \& Panza, J. A. (1998). Impaired endothelial regulation of vascular tone in patients with systemic arterial hypertension. Vascular Medicine, 3, 138-144.

Carrol, D., Ring, C., Hunt, K., Ford, G., \& Macintyre, S. (2003). Blood pressure reactions to stress and the prediction of future blood pressure: Effects of sex, age, and socioeconomic position. Psychosomatic Medicine, 65(6), 1058-1064.

De Wardener, H. E. (2001). The hypothalamus and hypertension. Physiological Reviews, 81(4), 1599-1658.

Dean, H., Celis, H., Vandenhoven, G., O’Brien, E., \& Staessen, J. A. (2003). Determinants of white-coat syndrome assessed by ambulatory blood pressure or self-measured home blood pressure. Blood Pressure Monitoring, 8(1), 37-40.

Devés, R., \& Boyd, C. A. (1998). Transporters for cationic amino acids in animal cells: Discovery, structure, and function. Physiological Reviews, 78, 487-545.

Dressler, W. W., \& Santos, J. E. (2000). Dimensões culturais e sociais da hipertensão no Brasil: Uma revisão. Cadernos de Saúde Pública (Rio de Janeiro), 16(2), 303-315.

Dubei, R. K., Boegehold, M. A., Gillespie, D. G., \& Rosseli, M. (1996). Increased nitric oxide activity in early renovascular hypertension. American Journal of Physiology, 39, R118-R124.

Esch, T., Stefano, G. B., Fricchione, G. L., \& Benson, H. (2002). Stress-related diseases - A potential role for nitric oxide. Medical Science Monitor, 8(6), RA103-RA118.

Eskiocak, S., Gozen, A. S., Taskiran, A., Kilic, A. S., Eskiocak, M., \& Gulen, S. (2006). Effect of psychological stress on the L-aginine-nitric oxide pathway and semen quality. Brazilian Journal of Medical and Biological Research, 39(5), 581-588.

Everly, G. S. (1989). A clinical guide to the treatment of the human stress response. New York: Plenum Press.

Givalois, L., Li, S., \& Pelletier, G. (2002). Central nitric oxide regulation of the hypothalamic-pituitary-adrenocortical axis in adult male rats. Brain Research. Molecular Brain Research, 102(1/2), 1-8.

Gulati, K., Ray, A., Masood, A., \& Vijayan, V. K. (2006). Involvement of nitric oxide (NO) in the regulation of stress susceptibility and adaptation in rats. Indian Journal of Experimental Biology, 44(10), 809-815.

Harmann, M., Dias-da-Costa, J. S., Olinto, M. T. A., Pattussi, M. P., \& Tramontini, A. (2007). Prevalência de hipertensão arterial sistêmica e fatores associados: Um estudo de base populacional em mulheres no Sul do Brasil. Cadernos de Saúde Pública (Rio de Janeiro), 23(8), 1857-1866.

Hashiguchi, H., Ross-Cisneros, F., \& Alexander N. (1997). Central nitric oxide donors attenuate cardiovascular and central norepinephrine responses to stress. American Journal of Physiology, 272(5, Pt. 2), 1447-1453. 
Malagris, L. M. N., Brunini, T. M. C., Moss, M. B., Silva, P. J. A., Esposito, B. R. \& Ribeiro, A. C. M. (2009). Evidências Biológicas do Treino de Controle do Stress em Pacientes com Hipertensão.

Holm, T., Aukrust, P., \& Aagaard, A. K. (2002). Hypertension in relation to nitric oxide, asymmetric dimetylarginine and inflammation: Different patterns in heart transplant recipients and individuals with essential hypertension. Transplantation, 74(10), 1395-1400.

Ishizuka, Y., Ishida, Y., Jin, Q., Kato, K., Kunitake, T., Mitsuyama, Y., et al. (2000). Differential profiles of nitric oxide and norepinephrine releases in the paraventricular nucleus region in response to mild footshock in rats. Brain Research, $862,17-25$.

Joint National Committee on Prevention, Detection, Evaluation and Treatment of High Blood Pressure. (2003). Report The Seventh Report of Joint National Committee on Prevention, Detection, Evaluation and Treatment of High Blood Pressure (JNC 7). JAMA, 289(19), 2560-2572.

Kakuda, D. K., Finley, K. D., Maruyama, M., \& MacLeod, C. L. (1998). Stress differentially induces cationic amino acid transporter gene expression. Biochimica et Biophysica ACTA, 1414, 75-84.

Kivimaki, M., Kinnunem, M. L., Pitkanen, T., Vahtera, J., Elovainio, M., \& Pulkkinen, L. (2004). Contribution of early and adult factors tosocioeconomic variation in blood pressure: Thirty-four-year follow-up study of school children. Psychosomatic Medicine, 66(2), 184-189.

Klahr, S. (2001). The role of nitric oxide in hypertension and renal disease progression. Nephrology Dialysis Transplantation, 16(Suppl. 1), 60-62.

Lantelme, P., \& Milonm, H. (2000). White coat hypertension. Archives des Maladies du Coeur et des Vaisseux, 93(12), 15341540.

Lauer, T., Kleinbongard, P., \& Kelm, M. (2002). Indexes of NO biovailability in human blood. News in Physiological Sciences, 17, 252-255.

Leonard, B. E. (2005). The HPA and immune axes in stress: The involvement of the serotonergic system. European Psychiatry, 20(3), 302-306.

Lipp, M. E. N. (1991). O valor do controle do stress como terapêutica da hipertensão arterial In M. Nobel (Ed.), Psicossomática (pp. 86-163). Campinas, SP: NEP.

Lipp, M. E. N. (2000). Inventário de Sintomas de Stress de Lipp. São Paulo, SP: Casa do Psicólogo.

Lipp, M. E. N. (2003a). O modelo quadrifásico do stress. In M. Lipp (Ed.), Mecanismos neuropsicofisiológicos do stress: Teoria e aplicações clínicas (pp. 17-21). São Paulo, SP: Casa do Psicólogo.

Lipp, M. E. N. (2003b). O tratamento psicológico do stress. In M. Lipp (Ed.), Mecanismos neuropsicofisiológicos do stress: Teoria e aplicações clínicas (pp. 187-192). São Paulo, SP: Casa do Psicólogo.

Lipp, M. E. N. (2004). O diagnóstico do stress em adultos. In M. Lipp (Ed.), Stress no Brasil: Pesquisas avançadas (pp. 5358). Campinas, SP: Papirus.

Lipp, M. E. N. (2005). Stress no trabalho: Implicações para a pessoa e para a empresa. In F. Nunes \& I. Nassaralla, Pedagogia institucional: Fatores humanos nas organizações (pp. 214236). Rio de Janeiro, RJ: Zit.

Lipp, M. E. N., Bignotto, M. M., \& Alcino, A. B. (1997). Efeitos do treino de controle do stress social na reatividade cardiovascular de pacientes hipertensos. Teoria, Investigação e Prática, 2(1), 137-146.

Lipp, M. E. N., \& Malagris, L. E. N. (1995). Manejo do estresse. In B. Rangé (Ed.), Psicoterapia comportamental e cognitiva: Pesquisa, prática, aplicações e problemas (pp. 279-292). Campinas, SP: Editorial Psy.
Lipp, M. E. N., \& Malagris, L. E. N. (2001). O stress emocional e seu tratamento. In B. Rangé (Ed.), Psicoterapias cognitivocomportamentais: Um diálogo com a psiquiatria (pp. 475490). Porto Alegre, RS: Artmed.

Lipp, M. E. N., Pereira, M. M. B., Justo, A. P., \& Matos, T. M. G. (2006). Cardiovascular reactivity in hipertensives: Differential effect of expressing and inhibiting emotions. The Spanish Journal of Psychology, 9(2), 154-161.

Malagris, L. E. N. (2005). Burnout: O profissional em chamas. In F. Nunes \& I. Nassaralla, Pedagogia institucional: Fatores humanos nas organizações (pp. 196-213). Rio de Janeiro, RJ: Zit.

Malagris, L. E. N., \& Fiorito, A. C. C. (2006). Avaliação do nível de stress de técnicos da área de saúde. Estudos de Psicologia (Campinas), 23(4), 391-398.

McEwen, B. S. (2003). Mood disorders and allostatic load. Biological Psychiatry, 54(3), 200-207.

Meireles, L. R., Mendes-Ribeiro, A. C., Santoro, M. M., Mendes, M. A. P., Silva, M. N. S. B., Mann, G. E., et al. (2007). The inhibitory effects of endogenous L-arginine analogues on nitric oxide synthesis in platelets: Role in platelet hyperaggregability in hypertension. Clinical and Experimental Pharmacology and Physiology, 34, 1267-1271.

Mendes Ribeiro, A. C., Hanssen, H., Kiessling, K., Roberts, N. B., Mann, G. E., \& Ellory, J. C. (1997). Transport of L-arginine and the nitric oxide inhibitor $\mathrm{N}^{\mathrm{G}}$-Monomethyl-L-arginine in human erythrocytes in chronic renal failure. Clinical Science, 93, 57-64.

Milakofsky, L., Harris, N., \& Vogel, W. H. (2000). Effects of repeated stress on plasma artinine levels in young and old rats. Physiology and Behavior, 54, 725-728.

Moncada, S., \& Higgs, E. A. (2006a). The discovery of nitric oxide and its role in vascular biology. British Journal of Pharmacology, 147(Suppl. 1), S193-S201.

Moncada, S., \& Higgs, E. A. (2006b). Nitric oxide and the vascular endothelium. Handbook of Experimental Pharmacology, 176, 213-254.

Moreno, H., Jr., Melo, S. E. S. F. C. S., \& Rocha, J. C. (2003). Stress e doenças cardiovasculares. In M. Lipp (Ed.), Mecanismos neuropsicofisiológicos do stress. Teoria e aplicações clínicas (pp. 99-105). São Paulo, SP: Casa do Psicólogo.

Moss, M. B., Brunini, M. C., Moura, R. S., Novaes Malagris, L. E., Robert, N. B., Ellory, C., et al. (2004). Dimished L-arginine bioavailability in hypertension. Clinical Science, 107, 391-397.

Pickering, T. G., \& Gerin, W. (1990). Cardiovascular reactivity in the laboratory and the role of behavioral factors in hypertension: A critical review. Annals of Behavioral Medicine, 12, 3-16.

Popovic, P. J., Zeh, H. J., III, \& Ochoa, J. B. (2007). Arginine and immunity. Journal of Nutrition, 137(6, Suppl. 2), 1681S$1686 \mathrm{~S}$.

Rudd, M. A., Trolliet, M. R., \& Loscalzo, J. (2000). Nitric oxide and hypertension. In J. Loscalzo \& J. A. Vita (Eds.), Nitric oxide and the cardiovascular system (pp. 227-246). Trenton, NJ: Human Press.

Selye, H. (1965). Stress: A tensão da vida. São Paulo, SP: IBRASA.

Simmons, W. W., Clossm, E. I., Cunningham, J. M., Smith, T. W., \& Kelly, R. A. (1996). Cytokines and insulin induce cationic amino acid transporter (CAT) expression in cardiac myocytes. Regulation of L-arginine transport and no production by CAT1, CAT-2A, and CAT-2B. Journal of Biological Chemistry, 271, 11694-11702. 
Sociedade Brasileira de Cardiologia., Sociedade Brasileira de Hipertensão., \& Sociedade Brasileira de Nefrologia. (2007). V Diretrizes Brasileiras de Hipertensão Arterial. Arquivos Brasileiros de Cardiologia, 89(3), e24-e79.

Stansfeld, S., \& Marmot, M. (2002). Introduction. In S. A. Stansfeld \& M. G. Marmot (Eds.), Stress and the heart: Psychosocial pathways to coronary heart disease (pp. 1-4). London: BMJ Books.

Straub, R. O. (2005). Psicologia da saúde. Porto Alegre, RS: Artmed.

Strike, P. C., \& Steptoe, A. (2004). Psychosocial factors in the development of coronary artery disease. Progress in Cardiovascular Diseases, 46(4), 337-347.

Taddei, S., Virdis, A. A., Mattei, P., Ghiadoni, L., Sudano, I., \& Salvetti, A. (1996). Defective L-arginine oxide pathway in offspring of essential hypertensive patients. Circulation, 84, 1298-1303.

Uji, M., Yoshida, K., Shintani, K., \& Morimoto, K. (2007). Sex difference in norepinephrine surge in response to psychological stress through nitric oxide in rats. Life Sciences, 80(9), 860866.

Vaziri, N. D., Ni, Z., \& Oveisi, F. (1998). Upregulation of renal and vascular nitric oxide sythase in young spontaneosusly hypertensive rats. Hypertension, 31(6), 1248-1254.

Verrey, F., Closs, E. I., Wagner, C. A, Palacin, M., Endou, H., \& Hanai, Y. (2004). CATs and HATs: The SLC7 family of amino acid transporters. Puflugers Archivers, 447, 532-542. 